\title{
Asociación del polimorfismo rs2241766 del gen de la adiponectina y enfermedad arterial coronaria en individuos del sur de Chile
}

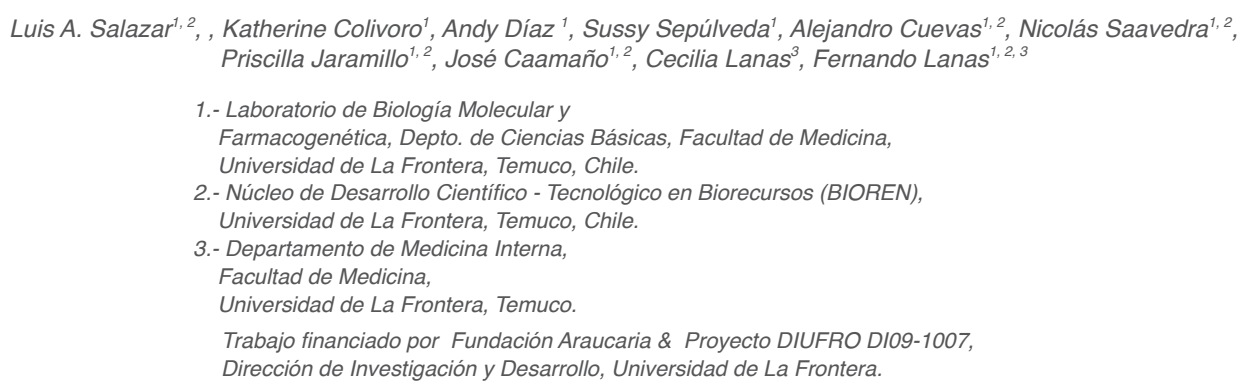

\section{Resumen:}

La adiponectina, hormona peptídica secretada por los adipocitos, actúa inhibiendo la formación de la placa ateromatosa en casi todas sus etapas, ya sea modulando la respuesta inflamatoria, inhibiendo la expresión de moléculas de adhesión, la activación de monocitos, la formación de células espumosas y la migración y proliferación de células de músculo liso. En el presente estudio, fue evaluada la frecuencia del polimorfismo $45 \mathrm{~T}>\mathrm{G}$ (rs2241766) del gen que codifica para la hormona adiponectina ( $A D I P O Q)$, junto con su posible contribución al desarrollo de enfermedad coronaria en individuos del sur de Chile.

Métodos: Fueron evaluados 416 individuos adultos
(30 a 68 años); 200 casos confirmados por angiografía y 216 controles. La genotipificación del polimorfismo $45 \mathrm{~T}>\mathrm{G}$ del gen $A D I P O Q$ se realizó mediante la técnica de PCR-RFLP.

Resultados: El análisis de los resultados demuestra que la presencia del alelo $\mathrm{G}$ del polimorfismo $45 \mathrm{~T}>\mathrm{G}$ del gen $A D I P O Q$ duplica el riesgo de padecer enfermedad coronaria en la población estudiada $(\mathrm{OR}=2.06$; I.C.95\%: 1.36 - 3.14).

Conclusión: Nuestros datos revelaron la existencia de una interesante asociación entre el polimorfismo $45 \mathrm{~T}>\mathrm{G}$ del gen $A D I P O Q$ y enfermedad arterial coronaria en los sujetos analizados. Es importante destacar, que este hallazgo constituye el primer antecedente en población chilena.

\section{Association between single nucleotide polymorphism of adiponectin gene and coronary artery disease in Southern Chilean subjects}

Background: Adiponectin, encoded by ADIPOQ gene, is a hormone secreted by adipocytes that acts inhibiting the atheromatous plaque formation, modulating the inflammatory response and inhibiting the expression of adhesion molecules with subsequent inhibition of adhesion and macrophage activation, foam cells formation and migration and proliferation of smooth muscle cells. Aim : to evaluate the possible association between the rs 2241766 polymorphism of the $A D I P O Q$ gene with coronary artery disease (CAD) in Southern Chilean subjects.

Methods: We evaluated 416 unrelated individuals (38 68 years old); 200 with CAD confirmed by angiography and 216 control individuals from Temuco city (Chile). The rs2241766 polymorphism $(45 \mathrm{~T}>\mathrm{G})$ of $A D I P O Q$ gene was determined by PCR-RFLP.

Results: CAD patients exhibited a high frequency of $\mathrm{G}$ allele when compared to controls ( $17 \%$ vs. $9 \%$; $p<0.001)$. The OR for CAD associated to $\mathrm{G}$ allele was 2.06 (95\%CI: 1.36 - 3.14) confirming the association observed.

Conclusion: Our data show that the rs2241766 ADIPOQ gene polymorphism contributed to $\mathrm{CAD}$ risk in the studied population.

Keywords: Coronary artery disease; Gene polymorphism; $A D I P O Q$.
Correspondencia:

Dr. Luis Antonio Salazar

Departamento de Ciencias Básicas, Facultad de Medicina,

Universidad de La Frontera.

Av. Francisco Salazar 01145, Casilla 54-D, Temuco

Fono: (45) 592895 - Fax: (45) 592832.

Correo Electrónico: Isalazar@ufro.cl 


\section{Introducción:}

Actualmente, el tejido adiposo es considerado un órgano endocrino, el cual está involucrado en diversos procesos fisiológicos ${ }^{1}$; posee una elevada actividad metabólica y como órgano endocrino es capaz de establecer comunicación con el resto del cuerpo mediante la síntesis y liberación de péptidos bioactivos llamados adipocitoquinas ${ }^{2}$.

Dentro de las adipocitoquinas secretadas por este tejido, tenemos a aquellas con función proinflamatoria: factor de necrosis tumoral alfa (TNF- $\alpha$ ), interleucina-6 (IL-6), inhibidor del activador del plasminógeno-1 (PAI-1) y angiotensinógeno, y aquellas con función endocrina: adipsina, leptina y adiponectina. Mediante la producción de estas moléculas, el tejido adiposo posee la capacidad de influenciar la biología local del adipocito y la del organismo ${ }^{3}$. Muchas de estas adipocitoquinas regulan el balance energético, las concentraciones de glucosa y el metabolismo lipídico, lo cual implica que su desregulación puede contribuir al desarrollo de las enfermedades asociadas a la obesidad ${ }^{4}$.

$\mathrm{La}$ adiponectina es una adipocitoquina únicamente expresada en el tejido adiposo blanco, y está presente en el plasma en importantes concentraciones (en promedio, $5-10 \mu \mathrm{g} / \mathrm{ml}$ ). Aunque el tejido adiposo es la única fuente de adiponectina, su concentración es, paradójicamente, reducida en la obesidad, en contraste con otras adipocitoquinas, tales como TNF-alfa, IL-6, PAI-1, leptina y resistina. Bajas concentraciones de adiponectina también han sido asociadas con insulinoresistencia, diabetes mellitus tipo 2 (DMT2) y aterosclerosis 5 .

En el proceso de aterosclerosis, la adiponectina actuaría inhibiendo la formación de la placa aterosclerótica en casi todas sus etapas, esto es, modulando la respuesta inflamatoria e inhibiendo la expresión de moléculas de adhesión, la adhesión y la activación de monocitos, la formación de células espumosas, y la migración y proliferación de células de músculo liso. Se han realizado estudios tanto in vitro como in vivo, donde se encontraron resultados similares; estableciéndose una relación directa entre los niveles de adiponectina y la reducción del riesgo a desarrollar un ateroma ${ }^{6}$. Se ha observado que las concentraciones plasmáticas de adiponectina son menores en individuos con enfermedad coronaria. También se encuentran disminuidos en sujetos con hipertensión, independientemente de la presencia de insulino-resistencia ${ }^{7}$.

El gen que codifica para la adiponectina $(A D I P O Q)$ está localizado en el cromosoma 3q27, en un locus susceptible para DMT2. Se han descrito varios polimorfismos de nucleótido simple (SNPs) en el gen $A D I$ $P O Q$; los más frecuentemente estudiados son $45 \mathrm{~T}>\mathrm{G}$ (rs2241766) y 276G $>\mathrm{T}$ (rs1501299), los cuales se han asociado a insulino-resistencia y a bajas concentraciones séricas de adiponectina ${ }^{8-10}$. También se ha encontrado asociación entre estos SNPs y la presencia de obesidad en individuos japoneses ${ }^{11}$. Sin embargo, otros autores han observado que la mutación I164T está asociada con enfermedad arterial coronaria e hipoadiponectinemia en población japonesa ${ }^{12}$. Otros polimorfismos, localizados en la región promotora del gen, tales como - $11391 \mathrm{G}>\mathrm{A}$ y $-11377 \mathrm{C}>\mathrm{G}$, han sido relacionados con bajos niveles séricos de adiponectina ${ }^{13,14}$. Sin embargo, no fueron asociados con insulino-resistencia y DMT2 en población francesa ${ }^{14}$.

Considerando las controversias mencionadas y el diferente origen étnico de nuestra población, el objetivo del presente estudio fue evaluar la posible asociación entre el polimorfismo 45T $>\mathrm{G}$ (rs2241766) del gen $A D I P O Q$ y la presencia de enfermedad coronaria en individuos del sur de nuestro país.

\section{Métodos}

\section{Sujetos}

El presente estudio, de tipo casos y controles, analizó un total de 416 individuos adultos. El grupo casos fue conformado por 200 individuos, de ambos sexos, con diagnóstico de enfermedad coronaria (historia y ECG de infarto al miocardio o enfermedad coronaria demostrada por angiografía con estenosis $>70 \%$ ), provenientes del Hospital Hernán Henríquez Aravena de la ciudad de Temuco. Por su parte el grupo control, fue integrado por 216 individuos sin enfermedad coronaria, sin parentesco, ni historia familiar de enfermedad cardiovascular precoz (parientes de primer grado $<55$ años para hombres y $<65$ años para mujeres), también pertenecientes a la región de La Araucanía.

A las personas que consintieron participar del estudio, se les aplicó un cuestionario sobre historia familiar de enfermedad cardiovascular, antecedentes mórbidos personales, entre otros. También se determinó peso, talla, pulso y presión arterial. Además, se obtuvieron dos muestras de sangre por punción venosa en ayunas, la primera sin anticoagulante, para determinaciones bioquímicas y la segunda, con EDTA para análisis molecular.

Este proyecto fue aprobado por el Comité de Ética 
Científica del Servicio de Salud Araucanía Sur. Todos los individuos que participaron de este estudio firmaron un Consentimiento Informado Escrito.

\section{Determinaciones bioquímicas}

Para las determinaciones de laboratorio se obtuvieron muestras de sangre venosa sin anticoagulante, previo ayuno de 12 horas. Las concentraciones séricas de colesterol total y triglicéridos fueron determinadas mediante métodos enzimático-colorimétricos; el colesterol HDL se midió previa precipitación selectiva de las LDL y VLDL con ácido fosfotúngstico e iones magnesio y posterior determinación por el método CHOD-PAP; la concentración de colesterol de las LDL fue calculada mediante la fórmula de Friedewald. Las concentraciones séricas de glucosa y ácido úrico fueron determinadas mediante métodos enzimáticocolorimétricos.

\section{Genotipificación de la variante $45 T>G$ del gen ADIPOQ}

El ADN genómico fue extraído a partir de sangre total anticoagulada con EDTA mediante la técnica de precipitación salina descrita por Salazar et al. ${ }^{15}$. Para genotipificar la variante $45 \mathrm{~T}>\mathrm{G}$ se amplificó un fragmento de 372 pares de bases (bp) mediante la reacción en cadena de la polimerasa (PCR) en un termociclador MyCycler (Bio-Rad Laboratorios, California, EE.UU.) usando los siguientes partidores: sense 5'GAAGTAGACTCTGCTGAGATGG-3' y antisense 3'- TATCAGTGTAGGAGGTCTGTGATG-5'. El protocolo de amplificación utilizado consideró para cada reacción: $200 \mathrm{nM}$ de cada primer, $0.2 \mathrm{nM}$ de dNTPs, $2 \mathrm{mM}$ de $\mathrm{MgCl}_{2}, 1$ U de Taq DNA Polimerasa (Fermentas, Lituania), 200 ng de ADN y agua destilada estéril, para un volumen final de $50 \mu 1$. La mezcla de reacción se sometió a una denaturación inicial a $98^{\circ} \mathrm{C}$ durante 3 minutos y 33 ciclos compuestos de denaturación a $95^{\circ} \mathrm{C}$, hibridación a $60^{\circ} \mathrm{C}$ y extensión a $72^{\circ} \mathrm{C}$, por 1 minuto cada fase, seguido de una extensión final a $72^{\circ} \mathrm{C}$ por 10 minutos. El producto amplificado fue evaluado en gel de agarosa al $1.5 \%$ teñido con bromuro de etidio $(0.5 \mathrm{mg} / \mathrm{L})$ y visualizado en transiluminador UV.

Posteriormente, los productos amplificados fueron sometidos a digestión enzimática usando la endonucleasa FastDigest ${ }^{\circledR}$ SmaI (Fermentas, Lituania) obtenida del microorganismo Serratia marcescen. En presencia del alelo $\mathrm{G}$, el segmento de $372 \mathrm{bp}$, es digerido resultando dos fragmentos de 219 y 153 bp. En cambio, en presencia del alelo $\mathrm{T}$, el sitio de reconocimiento para la enzima se pierde, por lo que no se evidencia digestión enzimática, obteniéndose un único fragmento de 372 bp. Para la restricción enzimática se utilizó una mezcla de reacción compuesta de $10 \mu 1$ de producto de PCR, $0.25 \mu 1$ de la endonucleasa (concentración 0.25 FDU), $2 \mu 1$ de FastDigest Buffer y agua destilada estéril hasta completar un volumen final de $20 \mu \mathrm{l}$, el cual fue incubado durante 40 minutos en baño termorregulado a $37^{\circ} \mathrm{C}$. Finalmente, estos fragmentos fueron evaluados mediante electroforesis en gel de agarosa al $2 \%$ teñido con bromuro de etidio.

\section{Control de calidad de las determinaciones \\ bioquímicas y moleculares}

La exactitud de las determinaciones bioquímicas fue controlada mediante la utilización de sueros comerciales normales y patológicos (Human, Alemania). La posibilidad de contaminación en los análisis moleculares fue excluida por la utilización de controles de reactivos en cada serie de amplificación. La correcta genotipificación del polimorfismo investigado fue confirmada mediante la repetición al azar del $20 \%$ de los análisis previamente realizados.

\section{Análisis estadístico}

Los datos obtenidos fueron analizados mediante la utilización del programa SigmaStat para Windows, versión 3.5. Inicialmente se hizo un análisis descriptivo a todas las variables. Las variables continuas se expresaron como media +/- desviación estándar. Se utilizó el test $t$ de Student para evaluar diferencias entre las variables analizadas. Además, se utilizó el test de Chi - cuadrado $\left(\mathrm{X}^{2}\right)$ para el análisis de las variables no continuas y para verificar el Equilibrio de Hardy - Weinberg. La Odds ratio (OR) y su respectivo intervalo de confianza de $95 \%$, asociada al alelo mutado $45 \mathrm{G}$ también fue calculada. En este estudio, un valor $\mathrm{p}<0,05$ fue considerado como estadísticamente significativo.

\section{Resultados:}

\section{Análisis de las características clínicas y de laboratorio}

Las características clínicas, antropométricas y de laboratorio de los sujetos en estudio son mostradas en la Tabla 1. Los individuos con EAC presentaron valores elevados de índice de masa corporal (IMC) y una mayor prevalencia de factores de riesgo tradicionales para EAC, incluyendo consumo de tabaco, diabetes, 


\begin{tabular}{|c|c|c|c|}
\hline & Casos (200) & Controles (216) & $\mathbf{P}^{*}$ \\
\hline Edad, a os & $57 \pm 10$ & $43 \pm 9$ & $<0.001$ \\
\hline Hombres, \% & 63 & 59 & 0.437 \\
\hline Diabetes, \% & 32 & 5 & $<0.001$ \\
\hline PAS, mm Hg & $140 \pm 23$ & $126 \pm 22$ & $<0.001$ \\
\hline $\mathrm{PAD}, \mathrm{mm} \mathrm{Hg}$ & $81 \pm 18$ & $76 \pm 9$ & 0.020 \\
\hline $\mathrm{IMC}, \mathrm{kg} / \mathrm{m} 2$ & $28.2 \pm 4.5$ & $25.8 \pm 4.5$ & $<0.001$ \\
\hline Fumadores, \% & 58 & 41 & $<0.001$ \\
\hline Hipercolesterolemia, \% & 79 & 20 & $<0.001$ \\
\hline Hipertensi n, \% & 77 & 25 & $<0.001$ \\
\hline Angina, \% & 82 & 0 & $<0.001$ \\
\hline IAM, \% & 67 & 0 & $<0.001$ \\
\hline Historia Familiar de EAC, \% & 33 & 0 & $<0.001$ \\
\hline \multicolumn{4}{|c|}{$\mathrm{N}$ mero de vasos obstruidos, \% } \\
\hline 1 & $28 \%$ & 0 & --- \\
\hline 2 & $24 \%$ & 0 & --- \\
\hline 3 & $48 \%$ & 0 & --- \\
\hline Colesterol total, mg/dL & $218 \pm 49$ & $173 \pm 31$ & $<0.001$ \\
\hline $\mathrm{LDL}-\mathrm{C}, \mathrm{mg} / \mathrm{dL}$ & $127 \pm 40$ & $94 \pm 25$ & $<0.001$ \\
\hline $\mathrm{HDL}-\mathrm{C}, \mathrm{mg} / \mathrm{dL}$ & $31 \pm 7$ & $50 \pm 11$ & $<0.001$ \\
\hline Triglic ridos, mg/dL & $184 \pm 173$ & $113 \pm 85$ & $<0.001$ \\
\hline Glucosa, mg/dL & $114 \pm 42$ & $93 \pm 36$ & $<0.001$ \\
\hline Acido rico, mg/dL & $5.6 \pm 1.6$ & $4.5 \pm 1.5$ & $<0.001$ \\
\hline
\end{tabular}

Tabla 1. Número de individuos en paréntesis; IAM; infarto agudo al miocardio; IMC, índice de masa corporal; EAC, enfermedad arterial coronaria; PAS, presión arterial sistólica; PAD, presión arterial diastólica; HDL-C, Colesterol de las HDL; LDL-C, Colesterol de las LDL. *Test t de Student o Chi-cuadrado.

hipertensión arterial, hipercolesterolemia e historia familiar de EAC $(\mathrm{P}<0.001)$. Las concentraciones de colesterol total, colesterol LDL, triglicéridos, glucosa y ácido úrico fueron más altas en los pacientes con EAC que en los controles $(\mathrm{P}<0.001)$. Además, los individuos con EAC presentaron niveles más bajos de HDL-C $(\mathrm{P}<0.001)$. Similarmente, se advirtió una diferencia significativa en los valores promedio de presión arterial sistólica $(\mathrm{P}<0.001)$ y diastólica $(\mathrm{P}=0.020)$ entre los grupos EAC y controles.

Análisis del polimorfismo 45T>G del gen ADIPOQ
La distribución genotípica y la frecuencia relativa de los alelos para el polimorfismo $45 \mathrm{~T}>\mathrm{G}$ del gen $A D I$ $P O Q$ es resumida en la Tabla 2. Se aprecia que en la distribución de genotipos, hay diferencias significativas entre los grupos estudiados $(\mathrm{p}=0.002)$; de igual forma, la frecuencia relativa de los alelos no fue semejante $(\mathrm{P}<0.001)$. En ambos grupos investigados, el alelo $\mathrm{T}$ fue más frecuente que el alelo $\mathrm{G}$, presentándose en $91 \%$ de los controles y en $83 \%$ de los casos. La odds ratio $(\mathrm{OR})$ para EAC relacionada al alelo mutado fue de 2.06 (I.C. 95\%: 1.36 - 3.14), confirmando la presencia de asociación. 


\section{Discusión:}

Estudios previos, le confieren a la adiponectina efectos antiinflamatorios y antiateroscleróticos ${ }^{6}$. Es conocido que variaciones genéticas podrían afectar las concentraciones séricas y/o actividad de esta hormona. Así, en el presente estudio, se investigó, por primera vez en nuestro país, la frecuencia del polimorfismo $45 \mathrm{~T}>\mathrm{G}$ del gen $A D I P O Q$, junto con su posible contribución al desarrollo de enfermedad coronaria en individuos de la región de La Araucanía.

El análisis de los parámetros clínicos y de laboratorio en ambos grupos estudiados, muestra que las concentraciones séricas de colesterol total, colesterol LDL, triglicéridos, glucosa y acido úrico fueron más altas en los sujetos con EAC. Además, las concentraciones de HDL-C fueron más bajas en este grupo; esos resultados confirman las conocidas asociaciones entre esos factores de riesgo tradicionales y enfermedad arterial coronaria $^{16,17}$.

El análisis molecular mostró que un 69\% de los individuos del grupo casos fueron homocigotos para el alelo normal (TT), un $28,5 \%$ heterocigotos (TG) y un $2,5 \%$ de homocigotos para la variante (GG). Mientras que en los controles, se observó un $82,3 \%$ de homocigotos para el alelo normal (TT), un $16.66 \%$ de heterocigotos (TG) y un $1.04 \%$ de homocigotos para la variante (GG). Además, la presencia del alelo $\mathrm{G}$ del polimorfismo $45 \mathrm{~T}>\mathrm{G}$ del gen $A D I P O Q$ fue asociado a 2.10 veces mayor riesgo enfermedad arterial coronaria.

En contraste con nuestros resultados, otros autores no han encontrado asociación entre el SNP 45T $>\mathrm{G}$ y enfermedad coronaria ${ }^{18}$. Se ha mencionado también que los SNPs $45 \mathrm{~T}>\mathrm{G}$ y $276 \mathrm{G}>\mathrm{T}$ del gen $A D I P O Q$ estarían asociados a diabetes tipo 2 y no tendrían ninguna influencia en los niveles plasmáticos de adiponectina en pacientes con enfermedad coronaria ${ }^{12}$. A pesar de esta controversia, todos los autores convergen en que el SNP 45T>G sí está asociado a uno u otro factor de riesgo que podría provocar el desarrollo de EAC.

Los resultados controversiales entre los diversos estudios, podrían deberse a las diferencias alélicas asociadas con el fenotipo de la enfermedad en distintas poblaciones. En Chile, no se han llevado a cabo estudios al respecto, por lo que este trabajo representa el primer reporte descrito en nuestro país, específicamente en individuos de la región de La Araucanía; zona donde existe mayor prevalencia de población de etnia amerindia, por lo cual no es posible comparar los resultados obtenidos con individuos caucásicos, japoneses, afro-

\begin{tabular}{|c|c|c|c|c|c|}
\hline & & Genoti & & Al & \\
\hline & TT & TG & GG & $\mathbf{T}$ & G \\
\hline $\begin{array}{l}\text { Casos } \\
(200)\end{array}$ & $\begin{array}{l}69 \% \\
(138)\end{array}$ & $\begin{array}{c}28.5 \% \\
(57)\end{array}$ & $\begin{array}{l}2.5 \% \\
\text { (5) }\end{array}$ & 0.83 & 0.17 \\
\hline $\begin{array}{l}\text { Controles } \\
\text { (216) }\end{array}$ & $\begin{array}{l}82.3 \% \\
(178)\end{array}$ & $\begin{array}{c}16.66 \% \\
(36)\end{array}$ & $\begin{array}{c}1.04 \% \\
(2)\end{array}$ & 0.91 & 0.09 \\
\hline & $x^{2}=\varsigma$ & l; $p=0$. & & $2^{2}=1$ & gl; p \\
\hline
\end{tabular}

Tabla 2. *Modelo aditivo, Número de individuos en paréntesis; gl, grados de libertad. Equilibrio de Hardy-Weinberg: Casos $\chi^{2}=0.096, p=0.756$; Controles $\chi^{2}=0.035 . p=0.850$ 
americanos, coreanos, entre otros; dada las diferencias étnicas, para los cuales si existen registros de estudios del SNP 45T > G del gen ADIPOQ en la literatura internacional.

El polimorfismo $45 \mathrm{~T}>\mathrm{G}$ del gen $A D I P O Q$ se produce por un cambio silencioso en el nucleótido 45 , de la base nitrogenada $\mathrm{T}$ por $\mathrm{G}$, lo que, sin embargo, no genera un cambio del aminoácido glicina, pese a la sustitución de ese nucleótido (GGT $\rightarrow$ GGG, Gly15Gly). La ubicación del cambio de base resulta muy cercana al límite exón - intrón, lo cual afecta a la maquinaria de splicing. Cada vez hay más evidencias, de que incluso las mutaciones silenciosas en la región codificante podrían modificar los niveles de RNAm, afectando el splicing y disminuyendo por lo tanto, la expresión de los genes. Reafirmando esta hipótesis, existen trabajos que han detectado bajos niveles de RNAm de adiponectina, en tejido adiposo humano, debido a la expresión diferencial del alelo específico relacionado con este $\mathrm{SNP}^{19}$. Como consecuencia, disminuye la cantidad de adiponectina, lo cual explica que menores niveles séricos de esta hormona incidan en el riesgo de EAC. Si bien el presente estudio, relaciona el SNP 45T $>$ G con EAC, también se ha encontrado en la literatura que la mayo- ría de los polimorfismos que afectan la expresión de una proteína, generalmente se encuentran asociados a otros polimorfismos de nucleótido simple o a mutaciones mediante desequilibrio de ligamiento. En el caso de SNPs que afectan al gen de la adiponectina, algunos estudios han investigado el efecto del haplotipo "TG", el cual asocia los alelos mutados para los polimorfismos $276 \mathrm{G}>\mathrm{T}$ y $45 \mathrm{~T}>\mathrm{G}$. Se ha observado que los individuos portadores del haplotipo TG presentan niveles más elevados de triglicéridos y HOMA-IR ${ }^{20}$.

En resumen, el presente estudio revela la existencia de una interesante asociación entre el SNP 45T $>\mathrm{G}$ del gen $A D I P O Q$ y enfermedad coronaria en individuos del sur de Chile. Sin embargo, esta observación necesita ser confirmada en forma independiente por otros grupos a nivel nacional, con el objeto de validar su utilización como herramienta de diagnóstico genético de riesgo de enfermedad coronaria. Además, es importante considerar para la ejecución de futuros estudios, una selección más rigurosa de los individuos controles. Idealmente, deberían incluirse individuos que no presenten lesiones, a los cuales se les realizó coronariografía por otros motivos (por ejemplo pases operatorios), y con edades similares a los casos.

\section{Referencias:}

1. SCHERER P. Adipose tissue, from lipid storage compartment to endocrine organ. Diabetes 2006; 55: $1537-$ 1545.

2. PEREZ M. Adipose tissue as an endocrine organ: pathophysiological and therapeutic implications. Rev Fac Med 2007; 15: 225-242.

3. LOZANO CO. Adipocitoquinas. Rev Endocrinol Nutr 2002; 10; 147-150.

4. FLIER JS. Obesity wars: molecular progress confronts an expanding epidemic. Cell 2004; 116:337-50.

5. SALMENNIEMI U, ZACHAROVA J, RUOTSALAINEN E, VAUHKONEN I, PIHLAJAMÄKI J, KAINU-
LAINEN S, et al. Association of adiponectin level and variants in the adiponectin gene with glucose metabolism, energy expenditure and cytokines in offspring of type 2 diabetic patients. J Clin Endocrinol Metab 2005; 90: 4216-4223.

6. PALOMER X, PEREZ A, BLANCO-VACA F. Adiponectina: un nuevo nexo entre obesidad, resistencia a la insulina y enfermedad cardiovascular. Med Clin (Barc). 2005; 124 : 388-95.

7. DOMINGUEZ RC. Adiponectina: El tejido adiposo más allá de la reserva inerte de energía. Rev Endocrinol Nutr 2007; 15:149-155.

8. SHIN MJ, JANG Y, KOH SJ, CHAE JS, KIM OY, LEE $\mathrm{JE}$, et al. The association of SNP276G $>\mathrm{T}$ at adiponectin gene with circulating adiponectin and insulin resistance in 
response to mild weight loss. Int J Obes (Lond) 2006; 30: 1702-1708

9. ZACHAROVA J, CHIASSON J, LAAKSO M, STOPNIDDM Study Group. The common polymorphisms (Single Nucleotide Polymorphism [SNP] +45 and SNP +276) of the adiponectin gene predict the conversion from impaired glucose tolerance to type 2. Diabetes 2005; 54: 893899.

10. MELISTAS L, MANTZOROS C, KONTOGIANNI M, ANTONOPOULOU S, ORDOVAS J, YIANNAKOURIS $\mathrm{N}$. Association of the $+45 \mathrm{~T}>\mathrm{G}$ and $+276 \mathrm{G}>\mathrm{T}$ polymorphisms in the adiponectin gene with insulin resistance in non-diabetic Greek women. Eur J Endocrinol 2009; 161:845-52.

11. NAKATANI K, NOMA K, NISHIOKA J, KASAI Y, MORIOKA K, KATSUKI A, et al. Adiponectin gene variation associates with the increasing risk of type 2 diabetes in non-diabetic Japanese subjects. Int J Mol Med 2005; 15:173-7.

12. OHASHI K, OUCHI N, KIHARA S, FUNAHASHI T, NAKAMURA T, SUMITSUJI S, et al. Adiponectin I164T mutation is associated with the metabolic syndrome and coronary artery disease. J Am Coll Cardiol 2003; 43:11951200 .

13. SCHWARZ PE, TOWERS GW, FISCHER S, GOVINDARAJALU S, SCHULZE J, BORNSTEIN SR, et al. Hypoadiponectinemia is associated with progression toward type 2 diabetes and genetic variation in the ADIPOQ gene promoter. Diabetes Care 2006; 29:1645-50.

14. VASSEUR F, HELBECQUE N, DINA C, LOBBENS S, DELANNOY V, GAGET $S$, et al. Single-nucleotide po- lymorphism haplotypes in the both proximal promoter and exon 3 of the APM1 gene modulate adipocyte-secreted adiponectin hormone concentrations and contribute to the genetic risk for type 2 diabetes in French Caucasians. Hum Mol Genet 2002; 11: 2607-2614.

15. SALAZAR LA, MELO C, CAVALLI S, HINUY H, HIRATA M, HIRATA RD. Micrométodo para la extracão de DNA genómico útil no diagnóstico molecular da hipercolesterolemia familiar. Rev Bras Anal Clin 2001; 33: 111116.

16. EXECUTIVE SUMMARY OF THE THIRD REPORT OF THE NATIONAL CHOLESTEROL EDUCATION PROGRAM (NCEP) Expert panel on detection, evaluation, and treatment of high blood cholesterol in adults (Adults treatment Panel III). J Am Med Assoc 2001; 285: 2486-97.

17. LANAS F. Factores de riesgo de Infarto en América Latina. Rev Chilena Cardiol 2007; 26: 227-230.

18. JUNG CH, RHEE E, KIM S, SHIN H, KIM B, SUNG K, et al. Associations between two single nucleotide polymorphisms of adiponectin gene and coronary artery diseases. Endocr J. 2006; 53: 671-677.

19. LACQUEMANT C, FROGUEL P, LOBBENS S, IZZO P, DINA C, RUIZ J. The adiponectin gene $\mathrm{SNP}+45$ is associated with coronary artery disease in type 2 (non-insulin dependent) diabetes mellitus. Diabet Med 2004; 21: 776781.

20. JANG Y, CHAE JS, KOH SJ, HYUN YJ, KIM JY, JEONG YJ, et al. The influence of the adiponectin gene on adiponectin concentrations and parameters of metabolic syndrome in non-diabetic Korean women. Clin Chim Acta 2008; 391: 85-90. 